Title: An Improved Thevenin Model of Lithium-ion Battery with High Accuracy for Electric Vehicles

Authors: Xiaofeng Ding, ${ }^{\text {a }}$, Donghuai Zhang ${ }^{\mathrm{a}}$, Jiawei Cheng ${ }^{\mathrm{a}}$, Binbin Wang ${ }^{\mathrm{a}}$, Patrick Chi kwong Luk ${ }^{b}$

Affiliation: ${ }^{a}$ School of Automation Science and Electrical Engineering, BeiHang University, Beijing 100191, China.

b The Power Engineering Centre, Cranfield University, Cranfield MK43 0AL, U.K.

Email: dingxiaofeng@buaa.edu.cn (X.F. Ding); zdh0011@buaa.edu.cn (D.H. Zhang); chengjiawei0218@126.com (J.W. Cheng); 16031023@buaa.edu.cn (B.B. Wang); p.c.k.luk@cranfield.ac.uk (P.C.K Luk).

*Corresponding Author: Xiaofeng Ding; Email: dingxiaofeng@buaa.edu.cn;

Telephone/Fax: +86 (10) 82339498. 


\title{
An Improved Thevenin Model of Lithium-ion Battery with High Accuracy for Electric Vehicles
}

\begin{abstract}
This paper proposes an improved Thevenin model of the Lithium-ion battery taking into account temperature influence on the calculation accuracy of the open circuit voltage of a battery. The calculation accuracy of the terminal voltage of a battery is improved without increasing the order of the model. Firstly, the model was proposed based on Thevenin model and the relationship between the open-circuit voltage and the state of charge. Then, based on the experimental results of the open-circuit voltage test and hybrid power pulse characteristic test, the parameters of the battery model were identified by polynomial fitting and genetic algorithm, respectively. Furthermore, the temperature effects were considered in both the open-circuit voltage and hybrid power pulse characteristic tests. Finally, the proposed model was tested and verified by experiments under the Dynamic Stress Test condition and the Urban Dynamometer Driving Schedule at different temperatures. The accuracy of the proposed model is high and the parameter identification error is less than $1 \%$.
\end{abstract}

Keywords: Lithium-ion power battery, Equivalent circuit model, Parameter identification 
Nomenclature

\begin{tabular}{|c|c|}
\hline$R_{0}$ & the ohmic internal resistance of the battery $(\Omega)$ \\
\hline$R_{\mathrm{Pi}}$ & the polarization internal resistance of the battery $(\Omega)$ \\
\hline$C_{\mathrm{Pi}}$ & the polarization internal capacitance of the battery $(\mathrm{F})$ \\
\hline$T$ & temperature $\left({ }^{\circ} \mathrm{C}\right)$ \\
\hline$U_{\mathrm{OC}}$ & the open circuit voltage of the battery (V) \\
\hline$U_{\mathrm{P}}$ & the resulting voltage drop on the polarization resistance $(\mathrm{V})$ \\
\hline$I_{\mathrm{L}}$ & the operation current $(\mathrm{A})$ \\
\hline$U_{\mathrm{t}}$ & the battery terminal voltage $(\mathrm{V})$ \\
\hline$\Delta t$ & the sampling interval $(\mathrm{s})$ \\
\hline$G A\left(x_{i}\right)$ & Individual $x_{i}$ fitness \\
\hline$g a_{\max }^{k}$ & The maximum objective function value of the generation $\mathrm{k}$ \\
\hline$g a\left(x_{i}\right)$ & The objective function value of individual $\boldsymbol{x}_{\boldsymbol{i}}$ \\
\hline$b^{k}$ & The selection offset value of individuals in generation $\mathrm{k}$ \\
\hline $\mathrm{N}$ & sampling number \\
\hline
\end{tabular}

Acronyms

\begin{tabular}{|c|c|}
\hline OCV & Open-Circuit Voltage \\
\hline SOC & State of Charge \\
\hline HPPC & Hybrid Power Pulse Characteristic \\
\hline DST & Dynamic Stress Test \\
\hline UDDS & Urban Dynamometer Driving Schedule \\
\hline EVs & Electric Vehicles \\
\hline SOT & State of Temperature \\
\hline SOH & State of Health \\
\hline SOP & State of Power \\
\hline PNGV & Partnership for a New Generation of Vehicles \\
\hline GNL & General Non-Linear \\
\hline
\end{tabular}




\begin{tabular}{|c|c|}
\hline DOD & Depths of Discharge \\
\hline GA & Genetic Algorithm \\
\hline
\end{tabular}




\section{Introduction}

With the development of economic globalization, energy security and energy economic have been given new meaning. Serious questions are also raised about its potential impacts on our environment . It has become a global consensus that a key solution to these challenges is to accelerate the development of Electric Vehicles (EVs). Lithium-ion battery, which is considered as a great development potential battery in electric vehicles, has key advantages including high nominal voltage, large specific energy and long life. Hence, it is widely used in EVs [1]. Since the lithium-ion batteries for EVs have high capacity and large number of serial-parallel circuits, coupled with such issues as safety, durability, uniformity and cost, battery management is vital. Battery management systems include thermal management, charge and discharge control, status diagnostics and protection mechanisms for EVs [2]. The battery management system can maintain the temperature of the battery pack between $20^{\circ} \mathrm{C}$ and $40^{\circ} \mathrm{C}$ through the heat dissipation system and heating assembly, so that the battery pack works optimally under different operating conditions [3]. Meanwhile it can control the charge and discharge of the battery between the batteries and the battery packs so that the State of Charge (SOC) can be quickly balanced to extend battery life and improve the endurance of EVs. At the same time, the voltage, current, temperature and other information of the battery pack can be fed back in real-time to avoid serious accidents such as battery thermal runaway and high voltage electric shock, resulting in more safe and reliable driving of the EVs.

The performance of lithium-ion batteries directly affects the long-term and stable operation of the energy storage system in EVs. An effective battery management system is needed to detect and diagnose the state of the lithium-ion battery in real-time. The state diagnostics of 
lithium-ion batteries includes: State of Charge (SOC), State of Temperature (SOT), State of Health (SOH) and State of Power (SOP) [4-6]. A battery model is the basis for SOC estimation, $\mathrm{SOH}$ evaluation and other performances analysis.

Therefore, many battery models that describe the battery performances have been established. In general, the models can be divided into two categories: electrochemical models and equivalent circuit models. The electrochemical models are generally based on the electrochemical reaction mechanism inside the battery. The parameters are determined by factors such as battery structure, materials and size, etc. Therefore, this structure of the model is complex, and the model parameters are difficult to calculate and determine, and it is not suitable for analysis based on simulation [7-9]. The equivalent circuit models are circuit topology models that use electrical components such as voltage sources, resistors, and capacitors to form a specific structure and network. Hence, the equivalent circuit models can also be seen as lumped parameter models, in which variables are independent of the spatial position and uniform throughout the models. It is suitable for simulation with related circuits. It can be used to simulate the dynamic characteristics of the battery more intuitively. Therefore, the equivalent circuit models are the more widely used [2], such as Rint model, Thevenin equivalent circuit model, second-order $R C$ model, Partnership for a New Generation of Vehicles (PNGV) model, General Non-Linear (GNL) model.

Rint model is a structure formed by connecting an ideal voltage source and an ohmic internal resistance in series. The structure is simple and has the smallest number of parameters. However, since the ohmic polarization and diffusion polarization are not considered, the Rint model has a low precision and it is rarely used in electric vehicles and other fields [10-12]. 
Thevinin model is also called the first-order model. Considering both the ohmic polarization and electrochemical polarization, the structure of the model is relatively simple and the calculation time is small. Hence, it has a good practical value. Under the condition that the SOH does not change significantly, the charging and discharging behavior of the lithium ion battery under the constant current and constant temperature condition can be simulated accurately. However, as the battery ages or the temperature changes greatly, the accuracy of the model will decrease [13].

The second-order RC model is based on the Thevenin model, which adds an additional $R C$ parallel model in series to a traditional Thevenin model. Since it takes into account ohmic polarization, electrochemical polarization and concentration polarization, the simulation results of the model is more accurate, but the structure is relatively complex and the computational time is longer [14-15]. The PNGV model is also a derivative model from the Thevenin model. A capacitor is connected in series on the main circuit of Thevenin model to describe the change of the open circuit voltage of the lithium ion battery [16-17]. GNL integrates the advantages of the above four models, with wider applicability and higher accuracy, while the structure is the most complex and the calculation time consumption is the largest [18].

From the above literature review it can be seen that the simple low-order models face the challenge of accurate characterization of the battery states, while complex high-order models will greatly increase the difficulty of calculation and identification of model parameters. It is noteworthy that so far temperature has not been taken into account in the aforementioned models. 
In [19], a novel model is proposed to predict the battery thermal runaway behaviors from kinetics analysis of cell components. The oven tests indicate that the model can credibly predict battery safety performance without assembling a real battery. In [20], a 3D electrothermal model is developed within the frame of open source computational fluid dynamics code OpenFOAM by coupling electric conduction with heat transfer and energy balance for a single lithium-ion cell. The model has reproduced well the evolution process of a cell from normal to abnormal cycling until thermal runaway. In [21], a system model of a stationary lithium-ion battery system is created for a use-case specific analysis of the system energy efficiency. The model offers a holistic approach by calculating conversion losses and auxiliary power consumption.

The above studies show thermal runaway models of lithium-ion batteries can improve their reliability when used in the EVs. Hence, in this paper, a new battery model considering temperature effects is proposed. The proposed battery model considers the influence of temperature and SOC on the Open-Circuit Voltage (OCV) without increasing the order of the model, and it is based on Thevenin model to improve the calculation accuracy of the battery terminal voltage.

The paper is organized as follows. Firstly, a novel model is proposed based on Thevenin model and the relationship between OCV and SOC. Then, the parameters of OCV model and RC are identified based on OCV tests and Hybrid Power Pulse Characteristic (HPPC) tests under different temperature conditions, respectively. Finally, the accuracy of the model and parameters are verified by experiments.

\section{Lithium-ion power battery modeling}


Based on the application characteristics of power battery management systems, the lumped parameter equivalent circuit models with circuit elements such as resistance and capacitance as the core have unique advantages in structure and precision. The typical equivalent circuit model consists of a voltage source, a resistor and n times RC network structures, which is referred to as an n-RC model, as shown in Fig. 1. The voltage source presents the OCV of the power battery, and the single resistance represents the ohmic internal resistance $R_{0}$ of the battery, which accounts for the contact resistance among the parts such as the electrode material, the diaphragm resistance, the electrolyte. Meanwhile, the $R C$ network is using polarization internal resistance $R_{\mathrm{Pi}}$ and capacitance $C_{\mathrm{Pi}}$ to reflect the dynamic characteristics such as the diffusion effect and the polarization effect of the battery.

By changing the number $\mathrm{n}$ in the model, that is, changing the number of RC networks, a battery equivalent circuit model of different orders can be constructed. Fig. 2 shows the Thevenin model, which can be regarded as a kind of $\mathrm{n}-R C$ model when $\mathrm{n}=1$, while $R$ int model mentioned above is a special case when $n=0$.

The Thevenin equivalent circuit model of the battery is a model based on the Thevenin's theorem. The model describes the internal polarization $R_{\mathrm{p} 1}$ and $C_{\mathrm{p} 1}$ of the battery and has strong dynamic adaptability. It can better reflect the dynamic performance of the battery, and the circuit structure is relatively simple as shown in Fig. 2. Besides, compared with the high-order $R C$ model shown in Fig.1, it is easier to calculate. The advantage of computation efficiency can be reflected in the shorter time of simulation and faster SOC equalization of battery. However, the Thevenin model does not consider the case where the battery OCV varies with SOC and temperature $T$. It can only represent the transient response of battery voltage 
precisely under a certain SOC value. Therefore, it is necessary to additionally consider using equation (1) to characterize the steady-state change of battery voltage as:

$$
U_{\mathrm{OC}}=f(\mathrm{SOC}, T)
$$

Equation (1) needs to model the OCV-SOC relationship through the OCV test of the battery, and the relationship is to be obtained by curve fitting. Then, the above model is combined with the first-order $R C$ model, namely Thevenin model, to create the new battery model as shown in Fig.3.

In Fig.3, $U_{\mathrm{OC}}$ is the open circuit voltage of the battery model, namely OCV, which is obtained by fitting the variable in $f(\mathrm{SOC}, T)$ and its parameters. $R_{0}$ is the equivalent ohmic internal resistance of the power battery. $R_{\mathrm{P}}$ is the polarization internal resistance. $U_{\mathrm{P}}$ is the resulting voltage drop on the polarization resistance $R_{\mathrm{P}} . C_{\mathrm{P}}$ is the battery polarization capacitance. $I_{\mathrm{L}}$ is the operation current. Normally, the sign of the battery current is negative when the battery is discharging, and positive when the battery is charging. $U_{\mathrm{t}}$ is the battery terminal voltage.

A specific calculation and analysis of the determined model is required, which can be derived from Kirchhoff's law as:

$$
\left\{\begin{array}{l}
U_{\mathrm{t}}=U_{\mathrm{OC}}-U_{\mathrm{p}}-I_{\mathrm{L}} R_{0} \\
I_{\mathrm{L}}=\frac{U_{\mathrm{p}}}{R_{\mathrm{p}}}+C_{\mathrm{p}} U_{\mathrm{p}} \\
U_{\mathrm{OC}}=f(S O C, T)
\end{array}\right.
$$

The mathematical relationship between the output voltage and the input current of the battery model can be deduced through the Laplace transform, and the result is shown in equation (3): 


$$
U_{\mathrm{t}}(s)=U_{\mathrm{OC}}(s)-I_{\mathrm{L}}(s)\left(R_{0}+\frac{R_{\mathrm{p}}}{1+R_{\mathrm{p}} C_{\mathrm{p}} \cdot s}\right)
$$

Then the transfer function of the model is:

$$
G(s)=\frac{U_{\mathrm{t}}(s)-U_{\mathrm{OC}}(s)}{I_{\mathrm{L}}(s)}=-\left(R_{0}+\frac{R_{\mathrm{p}}}{1+R_{\mathrm{p}} C_{\mathrm{p}} \cdot s}\right)=-\frac{R_{0}+R_{\mathrm{p}}+R_{\mathrm{p}} C_{\mathrm{p}} \cdot s}{1+R_{\mathrm{p}} C_{\mathrm{p}} \cdot s}
$$

The transfer function needs to be discretized. The simple replacement method, also known as the Euler method, is often used to map the system from the $s$-plane to the $Z$-plane. Euler method, as shown in equation (5), is used to map the equation based on the $s$-plane to the $Z$ plane for $z$ transformation as:

$$
s=\frac{1-z^{-1}}{\Delta t \cdot z^{-1}}
$$

where, $\Delta t$ is the sampling interval of the system, and $z^{-1}$ can be understood as the unit delay factor. Substituting equation (5) into equation (4) can deduce the equation based on the Zplane as:

$$
G\left(z^{-1}\right)=\frac{R_{0}+\left(\frac{R_{0}+R_{\mathrm{p}}}{R_{\mathrm{p}} C_{\mathrm{p}}} \Delta t-R_{0}\right) z^{-1}}{1+\left(\frac{\Delta t}{R_{\mathrm{p}} C_{\mathrm{p}}}-1\right) z^{-1}}
$$

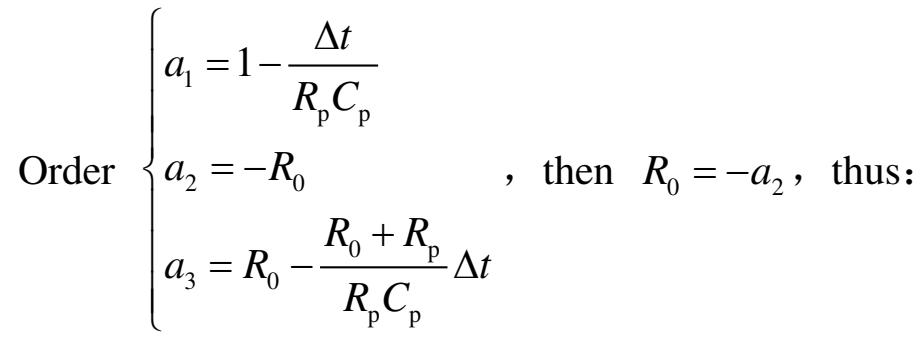

$$
\begin{aligned}
& G\left(z^{-1}\right)=\frac{U_{\mathrm{t}}\left(z^{-1}\right)-U_{\mathrm{OC}}\left(z^{-1}\right)}{I_{\mathrm{L}}\left(z^{-1}\right)}=\frac{a_{2}+a_{3} z^{-1}}{1-a_{1} z^{-1}}
\end{aligned}
$$

Then the corresponding difference equation is: 


$$
U_{\mathrm{t}, \mathrm{k}}-U_{\mathrm{OC}, \mathrm{k}}-a_{1}\left(U_{\mathrm{t}, \mathrm{k}-1}-U_{\mathrm{OC}, \mathrm{k}-1}\right)=a_{2} I_{\mathrm{L}, \mathrm{k}}+a_{3} I_{\mathrm{L}, \mathrm{k}-1}
$$

In order to simplify the calculation, considering the reasonable assumption that the actual sampling time interval is less than $1 \mathrm{~s}$, the change of the SOC value and the temperature of the power battery in the unit sampling time interval $\Delta t$ is approximately zero, hence, it can be considered that within the unit sampling interval $\Delta t$ the change of open circuit voltage is zero, that is:

$$
\Delta U_{\mathrm{OC}}=U_{\mathrm{OC}, \mathrm{k}}-U_{\mathrm{OC}, \mathrm{k}-1} \approx 0
$$

By substituting into equation (9), equation (8) can be simplified as follows:

$$
U_{\mathrm{t}, \mathrm{k}}=\left(1-a_{1}\right) U_{\mathrm{OC}, \mathrm{k}}+a_{1} U_{\mathrm{t}, \mathrm{k}-1}+a_{2} I_{\mathrm{L}, \mathrm{k}}+a_{3} I_{\mathrm{L}, \mathrm{k}-1}
$$

Equation (10) will be used for parameter identification of the battery model.

\section{Model and parameter identification}

\subsection{OCV model identification}

In order to analyze the open circuit voltage characteristics of the tested battery and get the relationship (1) between the OCV and the temperature $T$, it is necessary to identify the model based on the battery test results. Therefore, the OCV test of the battery is carried out first. This paper selects a model of ternary lithium battery with a capacity of $2 \mathrm{Ah}$, and experiments are undertaken with a battery test system. Considering the effect of temperature on the open circuit voltage, and OCV test needed to be done under different temperature conditions, it is necessary to use the thermostat to set the temperature condition, which can guarantee the temperature error within $\pm 1^{\circ} \mathrm{C}$. The battery pack is installed in the fixture and put it into the thermostat, and the TX77845 battery test system from Arbin Instrument is used 
to conduct the experiments. The experimental platform diagram is shown in Fig.4.

The test process is discharging the battery to the cut-off voltage (3V) first. After standing, it will charge $10 \%$ of the battery at a constant current rate of $0.5 \mathrm{C}$, it then stands for one hour each time. After the cut-off voltage $(4.1 \mathrm{~V})$ is reached, the battery is charged at a constant voltage until the current drops to $0.02 \mathrm{C}$ and then charging is stopped. After standing two hours without any charging or discharging action, it will then discharge $10 \%$ of the battery with a constant current $(0.5 \mathrm{C})$ and stand for one hour each time. When the battery is discharged to cut-off voltage, then the battery will stand for two hours. According to the battery product test specifications, the above steps are performed under the conditions of $10{ }^{\circ} \mathrm{C}, 25^{\circ} \mathrm{C}$ and $40{ }^{\circ} \mathrm{C}$, respectively. The test results are shown in Fig. 5 .

The OCV test results are processed. Specifically, the average value of the voltage measured in the charging process and discharging process is taken as the OCV of the battery, and the three-dimensional relationship scatter diagram of the OCV with respect to the SOC and the temperature $T$ can be obtained, as shown in Fig. 6 . The data points in the figure can be fitted to obtain the fitted surface of OCV with respect to SOC and $T$. And the results obtained by different fitting methods are also different. In order to obtain the model expression conveniently, the data is fitted by the polynomial method. In the fitting process, different orders are selected for the two variables $T$ and SOC respectively, and the fitting results are shown in Tab. 1.

Tab. 1 Polynomial fitting results

\begin{tabular}{ccccccc}
\hline & \multicolumn{5}{c}{ SOC Order } \\
Determination coefficient R-Square & 1 & 2 & 3 & 4 & 5 \\
\hline \multirow{2}{*}{$T$ Order } & 1 & 0.7960 & 0.8357 & 0.9410 & 0.9856 & 0.9959 \\
& 2 & 0.7970 & 0.8357 & 0.9410 & 0.9856 & 0.9960 \\
\hline
\end{tabular}


According to Tab. 1, the higher the order of SOC, the better the degree of fitting. When the order of SOC is 5 , the coefficient of determination is greater than 0.99 , which satisfies the fitting requirement, while the order of $T$ has little influence on the degree of fitting, and the difference is very small. Therefore, the model with the order of $T$ being 1 and the order of SOC being 5 is selected, and the detailed fitting results are shown in Fig. 7.

Then, the fitting results are substituted into equation (1) to obtain equation (11), which is the OCV model as follows:

$$
\begin{aligned}
& \mathrm{OCV}=3.276-0.0022 \cdot T+5.727 \cdot \mathrm{SOC}+0.0178 \cdot T \cdot \mathrm{SOC}-24.7 \cdot \mathrm{SOC}^{2} \\
& -0.0581 \cdot T \cdot \mathrm{SOC}^{2}+48.9 \cdot \mathrm{SOC}^{3}+0.0774 \cdot T \cdot \mathrm{SOC}^{3} \\
& -43.99 \cdot \mathrm{SOC}^{4}-0.0349 \cdot T \cdot \mathrm{SOC}^{4}+14.88 \cdot \mathrm{SOC}^{5}
\end{aligned}
$$

\subsection{HPPC test and RC parameter identification}

HPPC test is used to measure the pulse capability of batteries under different Depths of Discharge (DOD) in different time durations. It can be used for parameter identification of the battery model. HPPC tests at different temperatures are conducted to characterize the effects of temperature on battery characteristics. According to the battery product test specifications, the $\mathrm{HPPC}$ test process was carried out at $10^{\circ} \mathrm{C}, 25^{\circ} \mathrm{C}$ and $40^{\circ} \mathrm{C}$ respectively. And the power characteristics of SOC at 10 points from $100 \%$ to $10 \%$ were measured. The test results are shown in Fig. 8.

According to the obtained SOC curve and combined with the formula (11), the corresponding OCV value under different SOC and temperature can be calculated by the OCV model for parameter identification. In both engineering and scientific research fields, Genetic Algorithm (GA) is an effective method for parameter identification and optimization, which achieves the purpose of searching the optimal solution of the problem by simulating the natural 
evolution process. The flow chart of GA identification is shown as Fig.9.

The most important part of GA is to establish an appropriate fitness function through the objective function. Considering that the objective function is the minimum value, the dynamic linear calibration method is selected, as shown in equation (12):

$$
G A\left(x_{i}\right)=g a_{\max }^{k}-g a\left(x_{i}\right)+b^{k}
$$

where,

$G A\left(x_{i}\right)$ : Individual $x_{i}$ fitness;

$g a_{\max }^{k}:$ The maximum objective function value of the generation $\mathrm{k}$;

$g a\left(x_{i}\right)$ : The objective function value of individual $x_{i}$;

$b^{k}$ : The selection offset value of individuals in generation $\mathrm{k}$.

The addition of $b^{k}$ makes the worst individuals still have the possibility of breeding, which causes the diversity of the population to be maintained within the wide-area search range at the beginning, while the selection pressure can be gradually increased in the iteration process. Hence, the convergence of the population can be maintained in the local search area. The offset value $b^{k}$ can be given according to equation (13). It is noted that $M$ and $c$ are given constant values, and the range of general $c$ is $[0.9,1]$.

$$
\left\{\begin{array}{l}
b^{0}=M \\
b^{k+1}=c \cdot b^{k}
\end{array}\right.
$$

The data matrix of the system is defined as:

$$
\varphi_{\mathrm{k}}=\left[\begin{array}{llll}
U_{\mathrm{OC}} & U_{\mathrm{t}, \mathrm{k}-1} & \mathrm{I}_{\mathrm{L}, \mathrm{k}} & \mathrm{I}_{\mathrm{L}, \mathrm{k}-1}
\end{array}\right]
$$

The parameter matrix of the system is defined as: 


$$
\theta=\left[\begin{array}{llll}
1-a_{1} & a_{1} & a_{2} & a_{3}
\end{array}\right]
$$

Formula (10) is converted to the standard form of least squares method:

$$
U_{\mathrm{t}, \mathrm{k}}=\varphi_{\mathrm{k}} \theta
$$

For data with sampling number $\mathrm{N}$ :

$$
\begin{aligned}
& \phi_{\mathrm{N}}=\left[\begin{array}{llllll}
\varphi_{1} & \varphi_{2} & \ldots & \varphi_{\mathrm{k}} & \ldots & \varphi_{\mathrm{N}}
\end{array}\right]^{T} \\
& U_{\mathrm{N}}=\left[\begin{array}{lllllll}
U_{\mathrm{t}, 1} & \mathrm{U}_{\mathrm{t}, 2} & \ldots & \mathrm{U}_{\mathrm{t}, \mathrm{k}} & \ldots & \mathrm{U}_{\mathrm{t}, \mathrm{N}}
\end{array}\right]^{T}
\end{aligned}
$$

Select the objective function $J(\theta)$ as:

$$
J(\theta)=\sum_{i=1}^{\mathrm{N}}\left(U_{\mathrm{t}, \mathrm{i}}-\varphi_{\mathrm{i}} \theta\right)^{2}=\left(U_{\mathrm{N}}-\phi_{\mathrm{N}} \theta\right)^{T}\left(U_{\mathrm{N}}-\phi_{\mathrm{N}} \theta\right)
$$

In the Genetic Algorithm Toolbox based on MATLAB, the $m$ file of fitness function is written with $J(\theta)=0$ as the objective function, the data is imported in the way of global variables, and various parameters in the Genetic Algorithm are selected for iterative operation.

According to the formula (17), the fitting precision of the terminal voltage, namely, the sum of the squares of the residuals is selected as the fitness function. Meanwhile, the numerical range can be deduced according to the definition of three parameters and added into the algorithm as a constraint condition to increase the speed of search convergence and shorten the algorithm time. In the process of operation, the population number of the genetic algorithm is set as 50 , the maximum number of iterations is set as 100 , the crossover probability is set as 0.8 , and the mutation probability is set as 0.2 . The determination range of the parameter values can be obtained gradually after repeated operations for different data segments, and then the constraint condition of parameter range can be narrowed down gradually to realize the accurate search from global to local. After that, the data simulation results when temperature is $25^{\circ} \mathrm{C}$ can be obtained as shown in Fig. 10. Therefore, the optimal individual calculated by the inverse 
is:

$$
\left\{\begin{array}{l}
a_{1}=1-\frac{\Delta t}{R_{\mathrm{p}} C_{\mathrm{p}}}=0.988 \\
a_{2}=-R_{0}=-0.042 \\
a_{3}=R_{0}-\frac{R_{0}+R_{\mathrm{p}}}{R_{\mathrm{p}} C_{\mathrm{p}}} \Delta t=0.041
\end{array}\right.
$$

Tab. 2 The RC parameter identification results of battery model

\begin{tabular}{cc}
\hline Parameter & Value \\
\hline$R_{0} / \Omega$ & 0.042 \\
$R_{\mathrm{p}} / \Omega$ & 0.041 \\
$C_{\mathrm{p}} / \mathrm{F}$ & 2016.129 \\
\hline
\end{tabular}

The data of $\mathrm{HPPC}$ test at $10^{\circ} \mathrm{C}$ and $40^{\circ} \mathrm{C}$ are used for identification, and the results are basically the same. Since the sampling interval in the genetic algorithm means the generation interval, the sampling interval $\Delta t$ is $1 \mathrm{~s}$. Hence, it can be calculated that the parameters of the battery model are shown in Tab. 2 .

\section{Model verification}

The established battery model and the parameters identified were used to measure the battery SOC and temperature $T$ under the Dynamic Stress Test (DST) condition and the Urban Dynamometer Driving Schedule (UDDS), and all data points were calculated one by one using the model obtained from the identification results. The identification results of the battery terminal voltage $U_{\mathrm{t}}$ were obtained and compare with the $U_{\mathrm{t}}$ measured data under the DSTUDDS condition to verify the accuracy of the model and parameters. The experimental platform is shown in Fig.4. The battery fixture is placed in the thermostat, and the current data of the battery pack under UDDS operating conditions is used to charge and discharge the 
battery by the TX77845 battery test system to check the accuracy of the model.

From the verification results, as shown in Fig.11, the calculated results of $U_{\mathrm{t}}$ model of battery terminal voltage under DST-UDDS condition are very close to the measured results. The maximum error is $0.026 \mathrm{~V}$, the relative error is $0.7 \%$, the average error is $0.00052 \mathrm{~V}$, and the average relative error is $0.014 \%$. Compared with the traditional Thevenin model, the estimation accuracy of the proposed model is much improved.

The above analysis shows that the method of fitting the open circuit voltage based on the binomial method and the parameter identification method based on genetic algorithm can ensure that the model achieve high-precision simulation of the dynamic characteristics and the terminal voltage of the battery. Hence, the model provide a good condition for improving the prediction accuracy of the equilibrium variables in the battery management system.

\section{Conclusions}

In this paper, a new accuracy battery model is proposed, which combines Thevenin equivalent model and an empirical formula of Open-Circuit Voltage test. And the model considers the steady state and transient characteristics of the battery. The parameters of the model were identified by experimental tests and mathematical algorithm, respectively. Furthermore, the model was tested and verified by experiments under the Dynamic Stress Test and the Urban Dynamometer Driving Schedule conditions. It is found that the accuracy of the proposed model is high and the parameter identification error is less than $1 \%$.

\section{Acknowledgement}


This work was supported in part by the National Natural Science Foundation of China under Project 51877006. P.C.K. Luk also would like to acknowledge the partial support from EPSRC through Grant Ref: EP/L001063/1.

\section{References}

[1] H. Rahimi-Eichi, U. Ojha, F. Baronti and M. Chow. Battery management system: an overview of its application in the smart grid and electric vehicles, IEEE Trans. Ind. Electron M, vol.7, no.4, pp.4-16, Jun.2013.

[2] L.G. Lu, X.B. Han, J.Q. Li, J.F. Hua and M. Ouyang. A review on the key issues for lithium-ion battery management in electric vehicles[J]. Journal of Power Sources, 2013, 226(none):272-288.

[3] Z.Y. Jiang, Z.G. Qu. Lithium-ion battery thermal management using heat pipe and phase change material during discharge-charge cycle: A comprehensive numerical study[J]. Applied Energy, 2019, 242:378-392.

[4] C. Forgez, D. Vinh Do, G. Friedrich and C. Forgez. Thermal modeling of a cylindrical lifepo4/graphite lithium-ion battery. Journal of Power Sources, 195(9), 2961-2968

[5] K.Soon Ng, Chin-Sien Moo and Yi-Ping Chen. Enhanced coulomb counting method for estimating state-of-charge and state-of-health of lithium-ion batteries. Applied Energy, 2009, 86 (9): 1506-1511.

[6] C.F. Zou, C. Manzie, D. Nei and A. G. Kallapur. Multi-time-scale observer design for stateof-charge and state-ofhealth of a lithium-ion battery. Journal of Power Sources, 2016, 335: 
121-130.

[7] J. Li, K. Adewuyi, N. Lotfi, R. G. Landers, and J. Park. A single particle model with chemical/mechanical degradation physics for lithium ion battery state of health (SOH) estimation. Applied Energy, 2018, 212:1178-1190.

[8] R. Xiong, L. Li and Z. Li. An electrochemical model based degradation state identification method of Lithium-ion battery for all-climate electric vehicles application. Applied Energy, 2018, 219:264-275.

[9] L. Zheng, L. Zhang and J. Zhu. Co-estimation of state-of-charge, capacity and resistance for lithium-ion batteries based on a high-fidelity electrochemical model. Applied Energy, 2016, 180:424-434.

[10]PLETT, and L. Gregory. Extended Kalman filtering for battery management systems of LiPB-based HEV battery packs. Part 1. Background. Journal of Power Sources, 2004, 134(2): 252-261.

[11]PLETT, and L. Gregory. Extended Kalman filtering for battery management systems of LiPB-based HEV battery packs Part 2. Modeling and identification. Journal of Power Sources, 2004, 134(2): 262-276.

[12]PLETT, and L. Gregory. Extended Kalman filtering for battery management systems of LiPB-based HEV battery packs Part 3. State and parameter estimation. Journal of Power Sources, 2004, 134(2): 277-292.

[13]S. Cho, H. Jeong, C. Han, S. Jin, J. H. Lim, and J. Oh. State-ofcharge estimation for lithium-ion batteries under various operating conditions using an equivalent circuit model. Computers and Chemical Engineering, 2012, 41:1-9. 
[14]W.H. Zhu, Y. Zhu, and B. J. Tatarchuk. A simplified equivalent circuit model for simulation of $\mathrm{Pb}$-acid batteries at load for energy storage application. Energy Conversion and Management, 2011, 52 (89): 2794-2799.

[15]B. Wu and B. Chen. Study the performance of battery models for hybrid electric vehicles. Ieee/asme, International Conference on Mechatronic and Embedded Systems and Applications. IEEE, 2014:1-6.

[16] Y. Yang, T.F. Tang, D.T. Qin and M.H. Hu. PNGV Equivalent Circuit Model and SOC Estimation Algorithm of Lithium Batteries for Electric Vehicle. Journal of System Simulation, 2012.

[17]H.W. He, R. Xiong, Fan and X. Jin. Evaluation of lithium-ion battery equivalent circuit models for state of charge estimation by an experimental approach. Energies, 2011, 4(4): $582-598$

[18]D.S. Yu and H. Chen. Analysis of the influences from battery impedance on Behaviors of Switched Reluctance Drive. International Conference on Advanced Mechatronic Systems. IEEE, 2013.

[19]D.S. Ren, X. Liu, X.N. Feng and L.G. Lu. Model-based thermal runaway prediction of lithium-ion batteries from kinetics analysis of cell components[J]. Applied Energy, 2018, 228:633-644.

[20]P. Ping, Q.S. Wang, Y.M. Chung and J. Wen. Modelling electro-thermal response of lithium-ion batteries from normal to abuse conditions[J]. Applied Energy, 2017, 205:1327-1344.

[21]Michael S, Maik N, Nam T and Holger H. Energy efficiency evaluation of a stationary 
lithium-ion battery container storage system via electro-thermal modeling and detailed component analysis [J]. Applied Energy, 2018, 15:211-229. 


\section{Figure Captions}

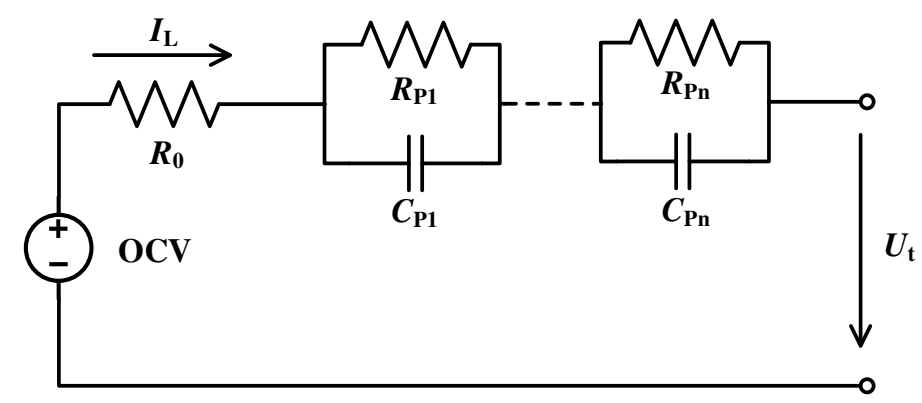

Fig. $1 \mathrm{n}-R C$ model

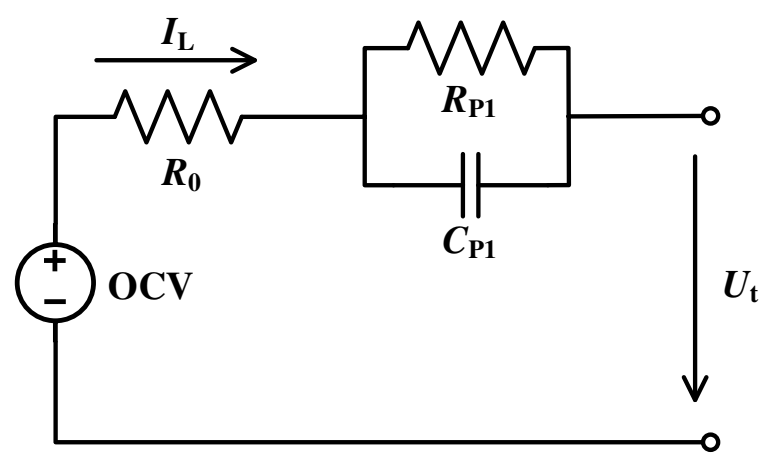

Fig. 2 Thevenin model

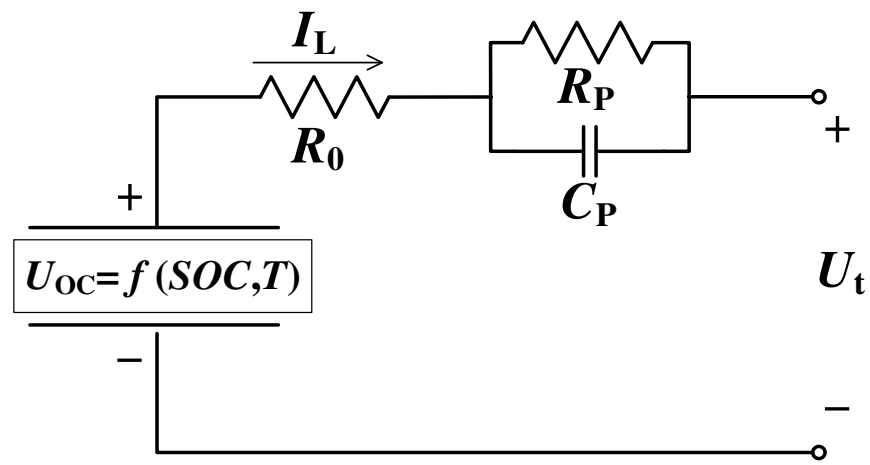

Fig. 3 The proposed battery model 


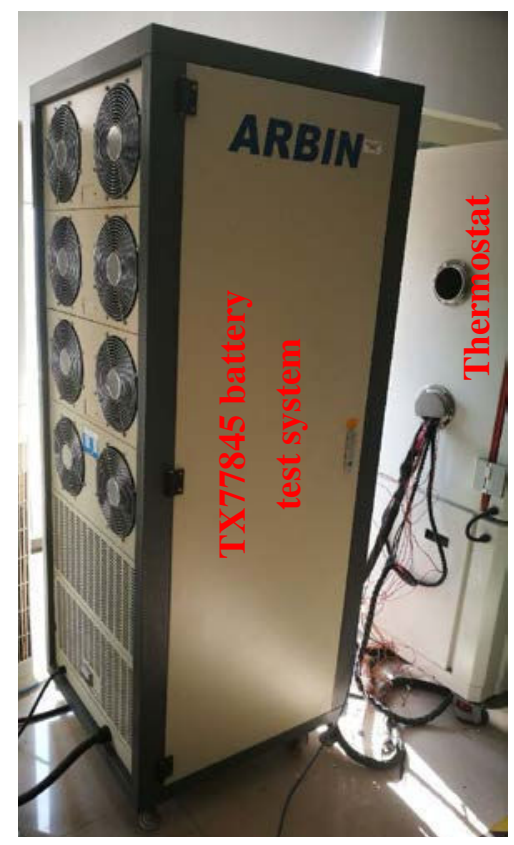

Fig.4. OCV test platform

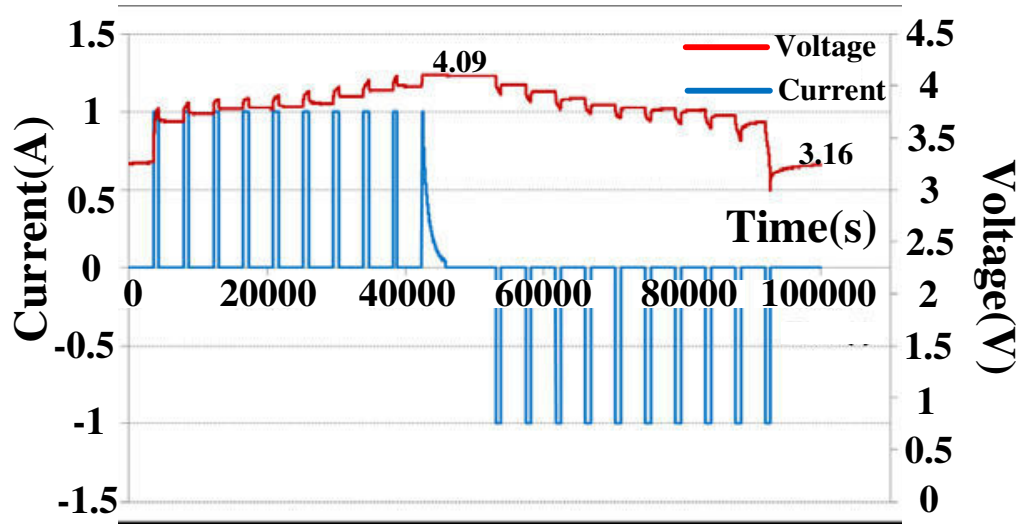

(a)

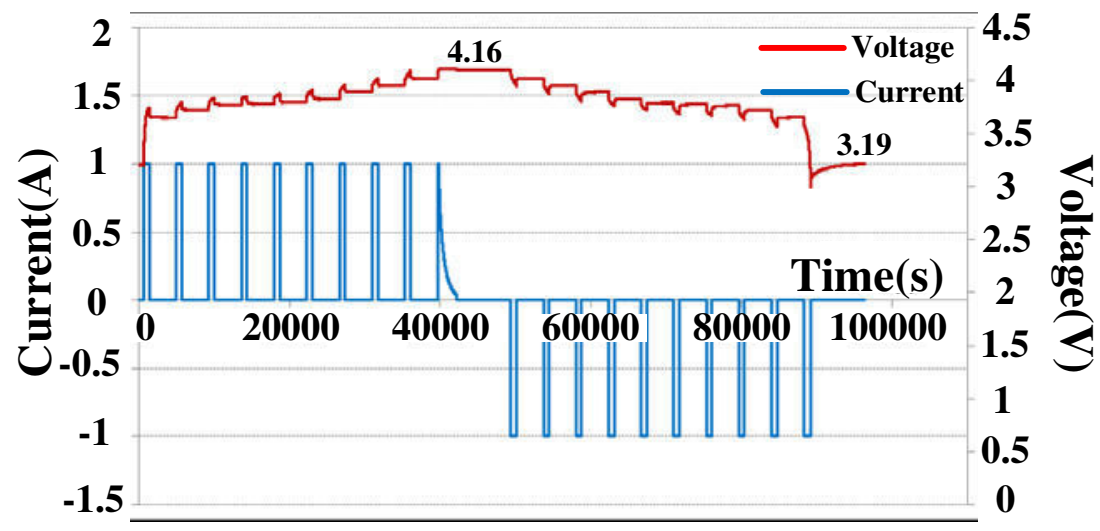

(b) 


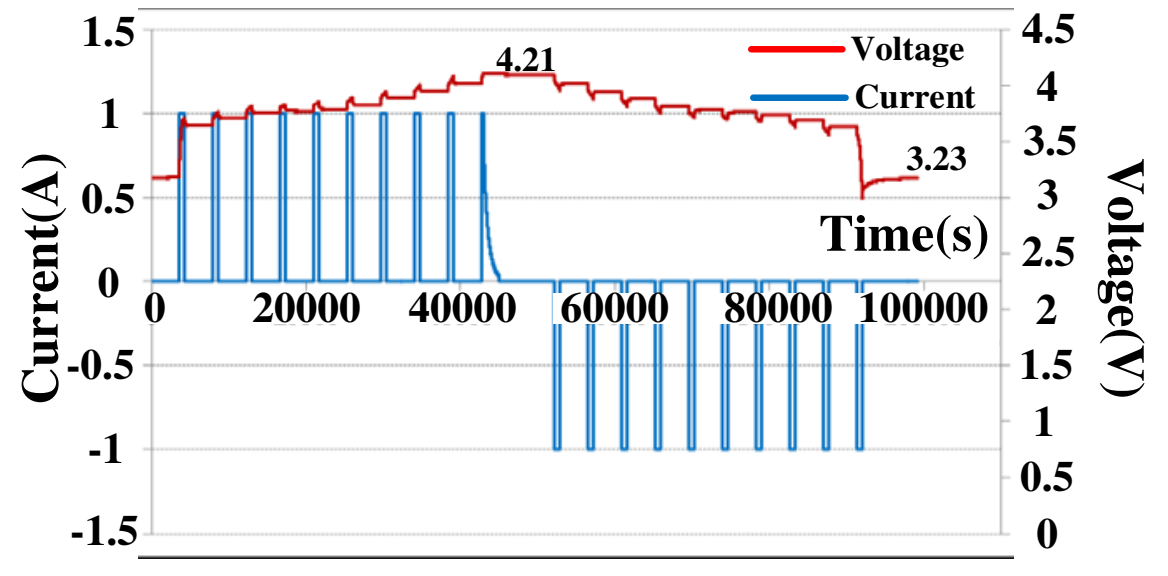

(c)

Fig. 5 Voltage and current curve under the OCV test with different temperatures: (a) $10^{\circ} \mathrm{C}$; (b) $25^{\circ} \mathrm{C}$; (c) $40^{\circ} \mathrm{C}$.

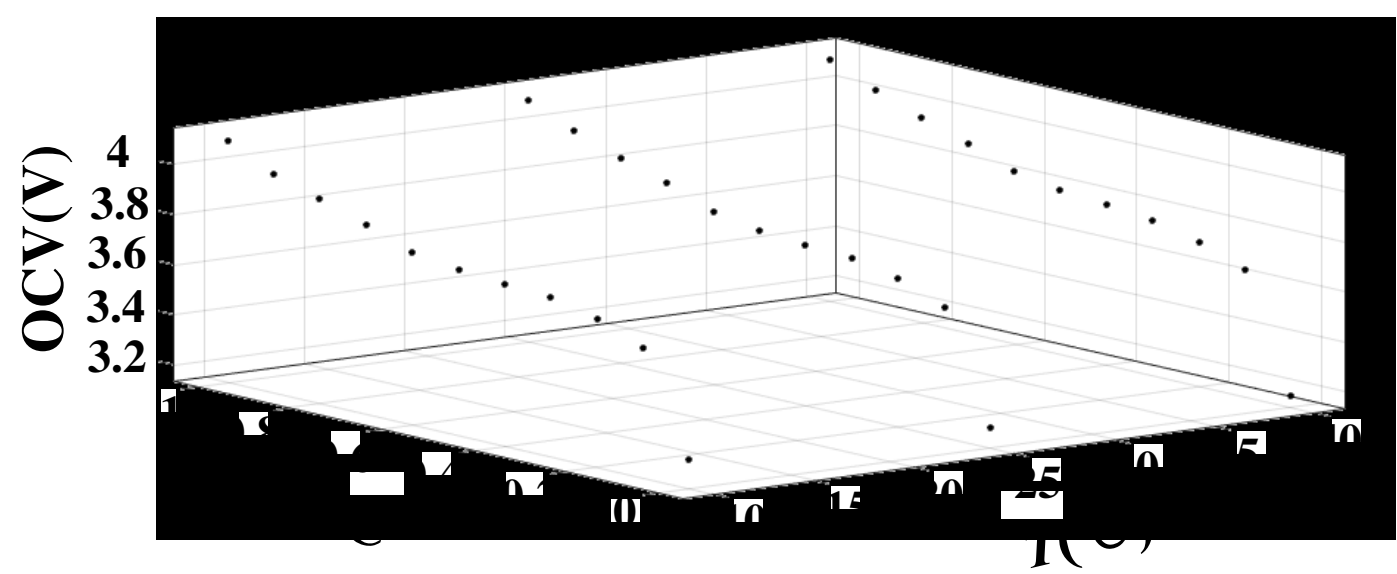

Fig. 6 Scatter plot of relationship between OCV and SOC, $T$

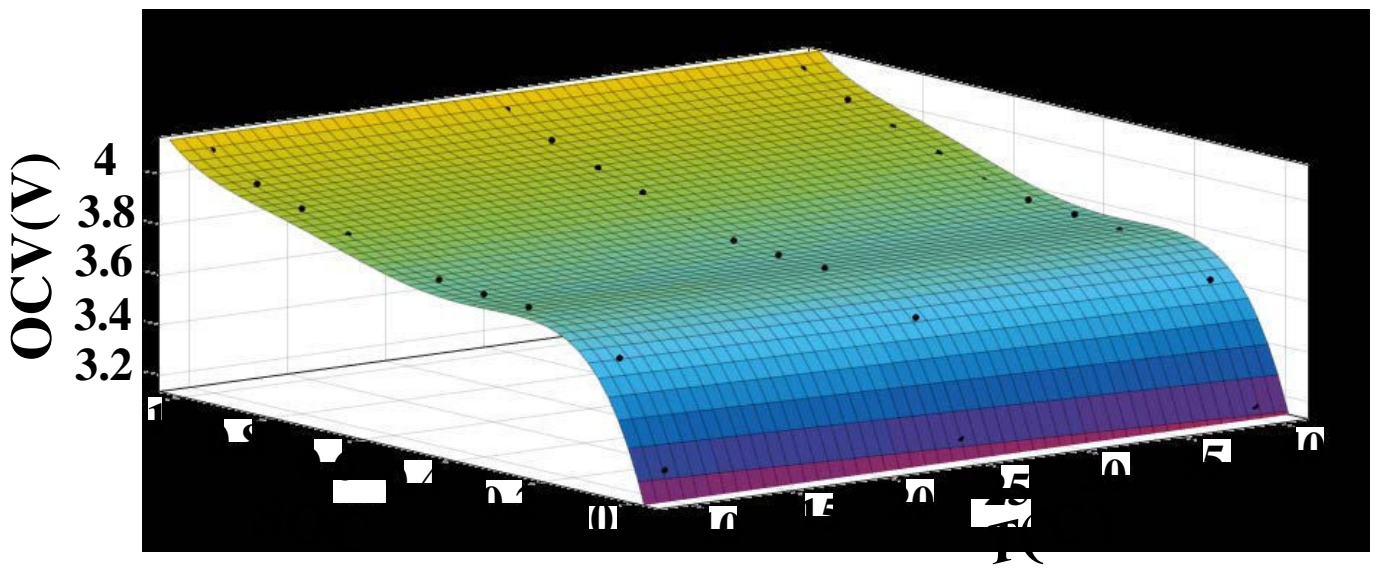

Fig. 7 OCV and SOC, $T$ relationship fit surface map 

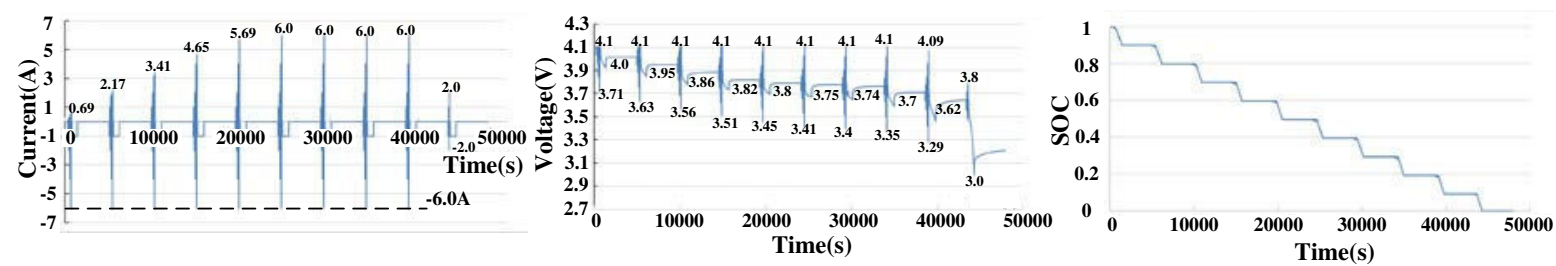

(a)
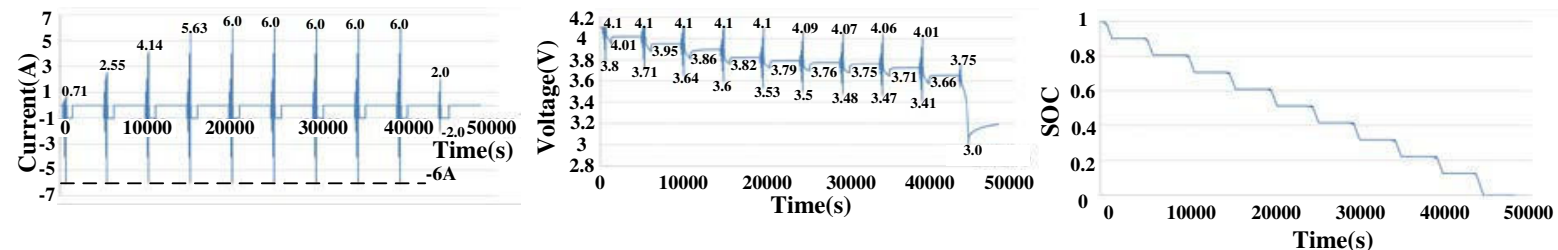

(b)
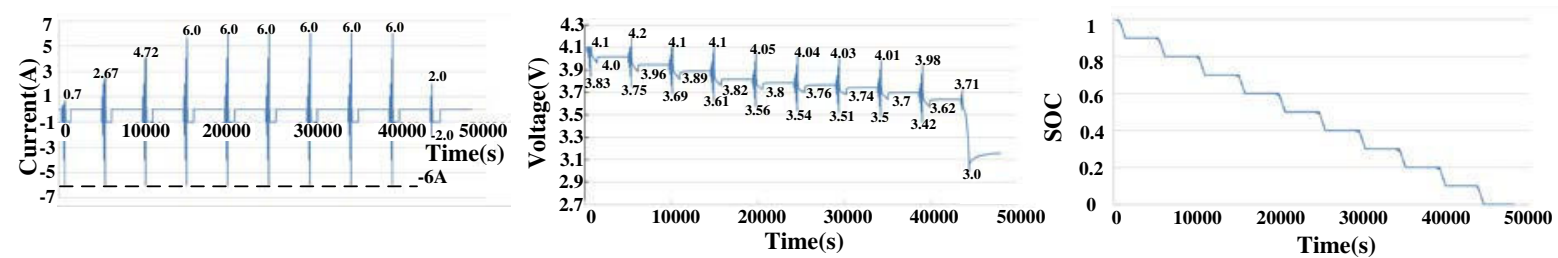

(c)

Fig. 8 HPPC test results under different temperatures: (a) $10^{\circ} \mathrm{C}$; (b) $25^{\circ} \mathrm{C}$; (c) $40^{\circ} \mathrm{C}$.

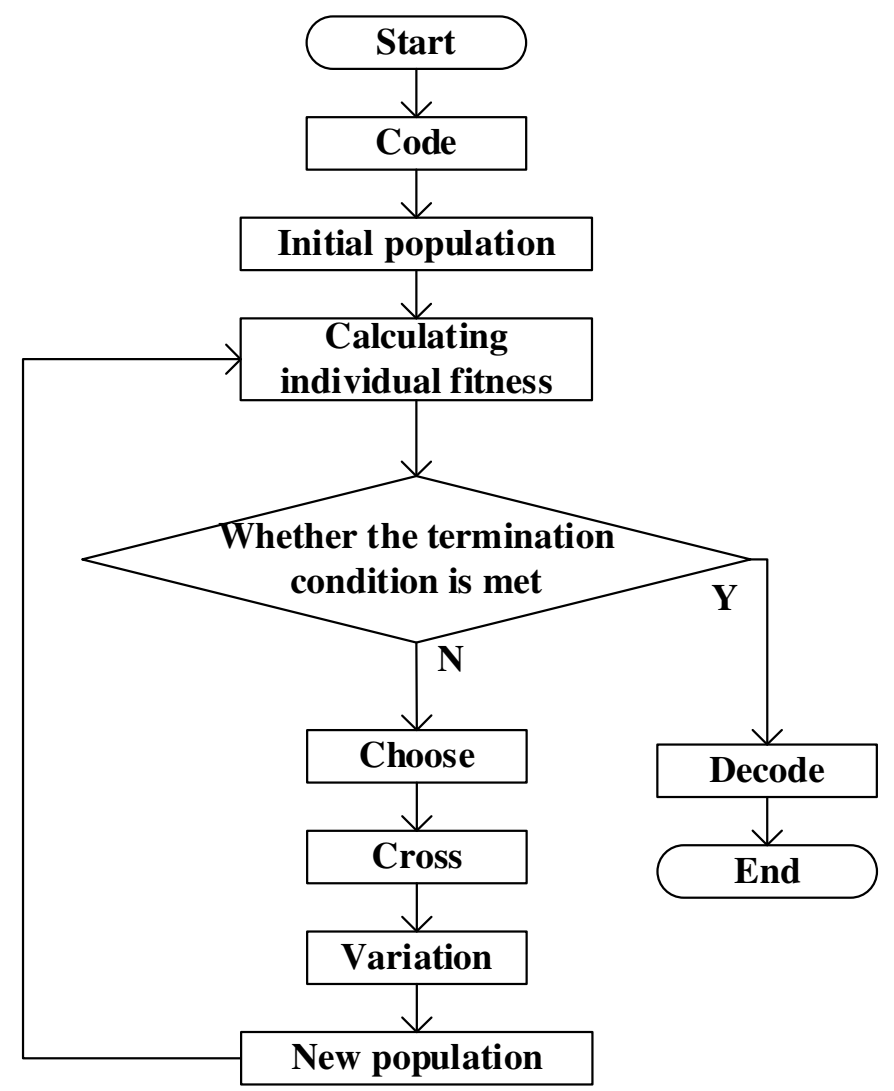

Fig.9 The flow chart of GA identification 

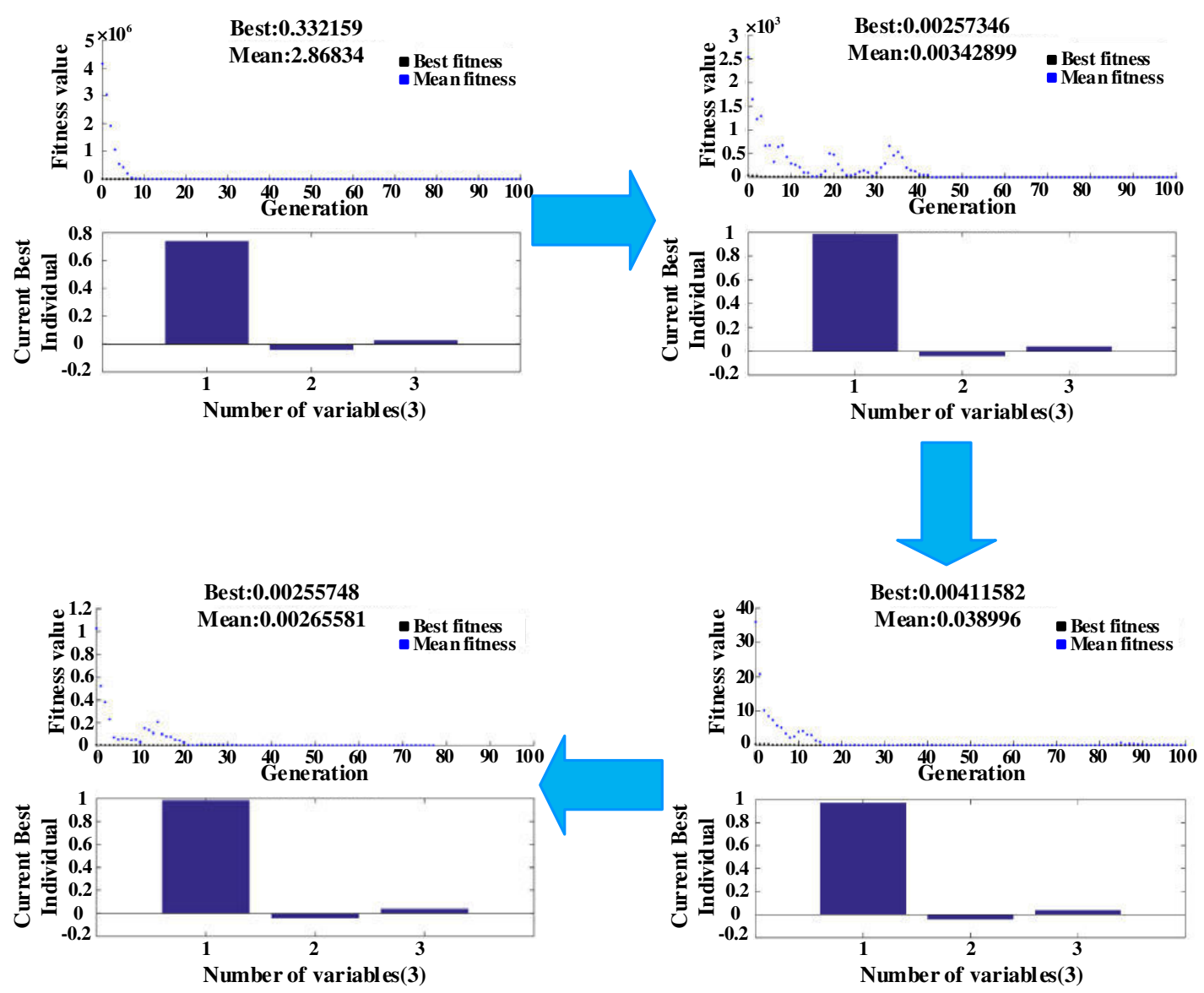

Fig. 10 Genetic algorithm search results

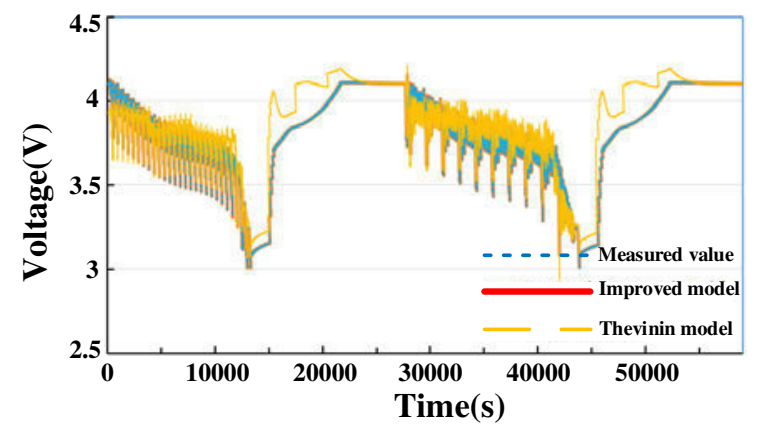

(a)

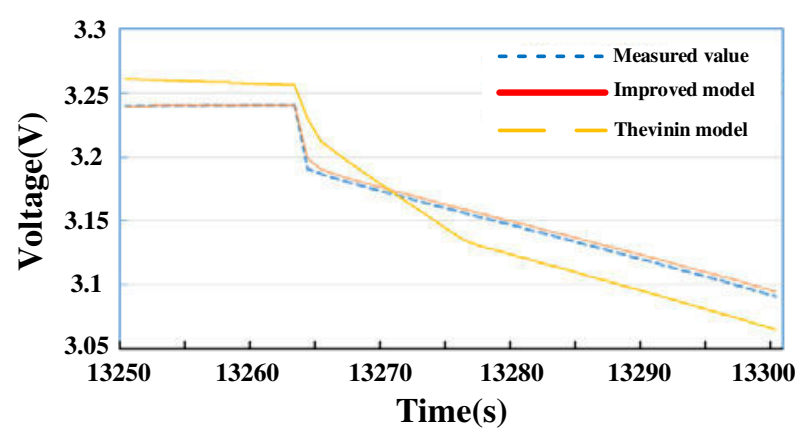

(b)

Fig. 11 Comparison of model verification results under DST-UDDS: (a) Full test; (b) Partial enlargement. 